\title{
Aerobic training increases skin perfusion by a nitric oxide mechanism in type 2 diabetes
}

This article was published in the following Dove Press journal:

Diabetes, Metabolic Syndrome and Obesity:Targets and Therapy

5 August 2010

Number of times this article has been viewed

\author{
Sheri R Colberg' \\ Laura C Hill ${ }^{2}$ \\ Henri K Parson ${ }^{3}$ \\ Kathleen S Thomas' \\ Aaron IVinik ${ }^{3}$
}

'Old Dominion University, Norfolk, Virginia, USA; ${ }^{2}$ State University of New York at Cortland, New York, USA; ${ }^{3}$ Eastern Virginia Medical School, Norfolk, Virginia, USA
Correspondence: Sheri Colberg

Human Movement Sciences Department, Old Dominion University, Norfolk,

VA 23529, USA

Tel + I 7576833356

Fax +I 7576834270

Email scolberg@odu.edu
Abstract: It is well known that a number of locally released vasodilatory and vasoconstrictive compounds can affect skin perfusion. This study investigated the effects of aerobic training on the contribution of nitric oxide (NO), prostaglandins (PG), and endothelial-derived hyperpolarizing factor (EDHF) in stimulated dorsal foot skin perfusion in individuals with type 2 diabetes (T2DM). Ten previously sedentary, older individuals with T2DM $(57.0 \pm 3.1$ years) and nine sedentary controls (53.5 \pm 3.2 years) were tested before and after undertaking six months of moderate aerobic training three times weekly in a supervised setting. All subjects underwent measurement of baseline $\left(32^{\circ} \mathrm{C}\right)$ and heat-stimulated $\left(40^{\circ} \mathrm{C}\right.$ and $\left.44^{\circ} \mathrm{C}\right)$ dorsal foot skin perfusion starting one hour after ingestion of a single, oral $325 \mathrm{mg}$ dose of aspirin, a known inhibitor of PG synthesis. Before aspirin ingestion, a subcutaneous microdialysis probe was inserted into each foot dorsum to administer either saline (PG pathway only blocked by aspirin in the left foot) or L-NAME (N(G)-nitro-1-arginine methyl ester; thereby inhibiting both PG and NO pathways in the right foot). Normative data collected previously on subjects undergoing saline administration via microdialysis without aspirin ingestion served as a control group. Significantly lower responsiveness of maximal perfusion was found with the EDHF pathway alone unblocked compared with $\mathrm{NO}$ and EDHF unblocked after training. Maximal suppression attributable directly to NO, PG, and EDHF was not significantly different when examined by subject group and training status. However, contributions of NO, PG, and EDHF to maximal perfusion were significantly increased, decreased, and unchanged by aerobic training, respectively, with diabetic and control subjects combined due to nonsignificant differences between groups. Improvements in maximally stimulated dorsal foot skin perfusion resulting from six months of aerobic training appear to have primarily an NO basis, with lesser contributions from PG following training, regardless of diabetes status.

Keywords: skin perfusion, aerobic training, type 2 diabetes, nitric oxide, prostaglandins, endothelial-derived hyperpolarizing factor, microdialysis

\section{Introduction}

It is well known that a number of locally released vasodilatory and vasoconstrictive compounds can affect skin perfusion. Among the vasodilators are nitric oxide (NO), prostacyclins, prostaglandins (PG), bradykinin, and endothelial-derived hyperpolarizing factor (EDHF), which appear to vary in importance depending on their cutaneal location. ${ }^{1-4}$ Delivery of nutrients, removal of metabolic waste, temperature regulation, and healing are all affected by maintenance of skin perfusion, but when type 2 diabetes mellitus (T2DM) has an underlying presence, alterations in foot skin perfusion may result in a greater risk of ulceration and poor cutaneous healing. ${ }^{3,5}$ 
Previously, it has been postulated that participation in chronic aerobic training (ie, regular exercise for longer than six months) improves stimulated dorsal foot skin perfusion through local release of vasodilatory compounds including NO, PG, and EDHF. ${ }^{5,6}$ A prospective study reported a significantly lower skin perfusion during local heating to $44^{\circ} \mathrm{C}$ in the dorsal feet of sedentary individuals with T2DM compared with active people without diabetes. ${ }^{6}$ This underlying defect was present despite normal increases in interstitial NO in the foot dorsum under stimulated conditions, suggesting that NO is either ineffective or not significantly involved in this acute response. ${ }^{3}$ A prior bout of acute maximal exercise also appears to improve responsiveness to local heating of the dorsal foot in active individuals with T2DM, but not in their sedentary counterparts. ${ }^{7}$

These previous studies have suggested that participation in chronic aerobic training may regulate stimulated (heated) dorsal foot skin perfusion through alterations in either PG or EDHF pathways, given that interstitial cutaneous NO levels (measured via a subcutaneous probe) are not apparently significantly depressed by the presence of T2DM. ${ }^{3,67}$ Prior research also suggests that chronic exercise participation (lasting a year or more) may change the relative importance of $\mathrm{PG}$ and EDHF in maximal dorsal foot skin perfusion during local heating more so than $\mathrm{NO} .^{8}$ Therefore, the purpose of this study was to investigate the chronic effect of relatively short-term (six months), moderate aerobic training on the relative importance of these skin vasodilators in previously sedentary, older individuals with and without T2DM.

\section{Methods}

\section{Subjects}

Ten sedentary T2DM and nine control subjects who were free of known cardiovascular disease, severe peripheral neuropathy, unstable proliferative retinopathy, end-stage renal disease, and uncontrolled hypertension participated in this study. In addition, no subjects were using insulin, angiotensin II receptor blockers, or angiotensin-converting inhibitors, due to their potential enhancing effects on nerve blood flow. ${ }^{9}$ Their sedentary status pretraining was verified using a modified version of the Harvard Alumni Physical Activity Questionnaire. ${ }^{6}$ By self-report, "sedentary" subjects had failed to engage in aerobic activities lasting 30 or more minutes at least three times per week for at least six months prior to the study.

After all subjects signed the informed consent approved by the Institutional Review Boards at both Eastern Virginia Medical School and Old Dominion University, they then underwent a history and physical examination, body fat estimation (via skin folds), exercise testing, and fasting blood work. Their resting and exercise characteristics are shown in the Table 1. Peripheral and autonomic neuropathy was assessed as previously described. ${ }^{10}$

\section{Aerobic exercise testing and training}

After undergoing cardiopulmonary exercise testing on a stationary cycle with measurement of peak oxygen consumption, subjects participated in moderate aerobic training for six months, consisting of three weekly sessions of 45 minutes of training at $50 \%-65 \%$ of heart rate reserve (ie, a set percentage of the difference between resting and maximal heart rate

Table I Subject characteristics

\begin{tabular}{|c|c|c|c|c|}
\hline & CON Pre & CON Post & DM Pre & DM Post \\
\hline $\begin{array}{l}\text { Subject number } \\
\text { (Male/Female) }\end{array}$ & $9(5 / 4)$ & & $10(3 / 7)$ & \\
\hline Age (years) & $57.0 \pm 3.1$ & & $53.5 \pm 3.2$ & \\
\hline Height $(\mathrm{cm})$ & $172.2 \pm 4.1$ & & $172.0 \pm 2.8$ & \\
\hline Weight (kg) & $89.9 \pm 13.6$ & $88.3 \pm 6.8$ & $99.1 \pm 8.0$ & $98.3 \pm 8.0$ \\
\hline Body fat $(H)$ & $32.4 \pm 4.3$ & $33.6 \pm 2.2$ & $37.6 \pm 2.2$ & $33.1 \pm 2.1$ \\
\hline Heart rate (bpm) & $71.1 \pm 2.8$ & $64.2 \pm 3.2$ & $82.3 \pm 1.8$ & $77.6 \pm 1.8$ \\
\hline Blood glucose (mM) & $4.6 \pm 0.1$ & $4.9 \pm 0.2$ & $7.1 \pm 0.1 *$ & $6.7 \pm 0.7^{\uparrow}$ \\
\hline Serum insulin $(\mu \mathrm{I} \mathrm{U} / \mathrm{ml})$ & $6.4 \pm 1.1$ & $6.4 \pm 1.5$ & $15.6 \pm 4.5^{*}$ & $10.9 \pm 0.8^{\uparrow}$ \\
\hline $\mathrm{HbA}_{\mathrm{lc}}(\%)$ & $5.9 \pm 0.2$ & $5.8 \pm 0.1$ & $7.3 \pm 0.3^{*}$ & $7.4 \pm 0.3^{\uparrow}$ \\
\hline $\mathrm{VO}_{2}$ peak $(\mathrm{mL} / \mathrm{kg} / \mathrm{min})$ & $19.0 \pm 2.2$ & $19.3 \pm 2.1$ & $17.1 \pm 1.3$ & $19.5 \pm 1.5$ \\
\hline E:I ratio & $1.16 \pm 0.02$ & $1.18 \pm 0.03$ & $1.15 \pm 0.03$ & $1.19 \pm 0.04$ \\
\hline Total Neuropathy Score & $4.1 \pm 1.0$ & $3.5 \pm 0.6$ & $4.1 \pm 0.7$ & $3.4 \pm 0.7$ \\
\hline
\end{tabular}

Notes: $* P<0.05$ versus controls pretraining; ${ }^{\uparrow} P<0.05$ versus controls post-training.

Abbreviations: $\mathrm{CON}$, control subjects; DM, diabetic subjects; Pre, pre-training; Post, post-training; bpm, beats per minute; $\mathrm{HbA}_{\mathrm{Ic}}$, glycated hemoglobin; VO ${ }_{2}$ peak, peak oxygen consumption; E:I ratio, expiration-to-inspiration ratio. 
that is added to the resting value). All training sessions were supervised by exercise personnel and heart rate monitored to ensure that training sessions were completed in the prescribed target range. Subjects were allowed to choose among different modes of training, including treadmill walking, stationary cycling, elliptical strider, and seated rowing exercise. Subjects were required to undertake a chosen activity for a minimum of 10 minutes before another could be substituted during any given training session.

\section{Skin perfusion measurements}

Skin perfusion was measured using laser Doppler assessment with probes positioned on each mid-metatarsal foot dorsum both before and after completion of training. The latter was conducted 48-72 hours after completion of the last exercise session so that only chronic training effects were being measured rather than the residual effects of acute exercise. Baseline flow was assessed under the probe during sequential heating to $32^{\circ} \mathrm{C}$ for 50 minutes, $40^{\circ} \mathrm{C}$ for 10 minutes, and $44^{\circ} \mathrm{C}$ for 40 minutes to induce neurogenic vasodilation, ${ }^{4}$ starting about one hour after subcutaneous microdialysis probe insertion on each foot dorsum to administer L-NAME (N(G)-nitro-1-arginine methyl ester, right foot) or saline (left foot), as done previously. ${ }^{8}$

Briefly, a small dose of L-NAME a known NO synthase inhibitor, dissolved in $2 \mathrm{~mL}$ of normal saline, was administered over 100 minutes starting one hour after subjects ingested a single $325 \mathrm{mg}$ oral dose of generic aspirin to inhibit all but the EDHF pathway in the right foot and the PG pathway alone in the left foot. To prepare the infusate, $4 \mathrm{~mL}$ of sterile $9 \%$ saline were added to a single vial containing $220 \mathrm{mg}$ of L-NAME, and after mixing, $2 \mathrm{~mL}$ were withdrawn using the microsyringe. Infused at a rate of $2 \mu \mathrm{L}$ per minute over 100 minutes, about $200 \mu \mathrm{L}$ of the L-NAME solution was administered locally to a small area of skin under the laser Doppler probe by the end of the testing period.

Normative data collected previously on subjects undergoing saline administration via microdialysis without aspirin ingestion served as a control for this study. ${ }^{8}$ The normative value used to determine percent suppression was 150 arbitrary perfusion units during maximal stimulation caused by localized heating to $44^{\circ} \mathrm{C}$.

\section{Statistical analyses}

Separate repeated one-way analysis of variance (ANOVA) measures were employed to compare skin perfusion during each heating condition (ie, baseline at $32^{\circ} \mathrm{C}$, heating to $40^{\circ} \mathrm{C}$, heating to $44^{\circ} \mathrm{C}$ ) between groups (T2DM versus controls) both pre- and post-training. Pooled data with subject groups combined were analyzed with results from maximal heating to $44^{\circ} \mathrm{C}$ only. Resting subject characteristics (T2DM versus controls) were analyzed using unpaired t-tests. Significance for all statistical analyses was set at $P<0.05$.

\section{Results}

T2DM subjects had significantly higher fasting serum glucose, insulin, and glycated hemoglobin levels than controls both pre-and post-training $(P<0.05)$. Body fatness was similar between groups pre-training, as were resting heart rate, peak oxygen consumption, and neuropathy scores, and none of these measures were significantly altered by training.

No significant differences in skin perfusion under baseline $\left(32^{\circ} \mathrm{C}\right)$ conditions or at $40^{\circ} \mathrm{C}$ were observed (data not shown). With blockage of PG or PG and NO pathways during maximally stimulated conditions (local heating to $44^{\circ} \mathrm{C}$ ), significantly greater suppression of dorsal foot skin perfusion was observed with the non-PG, non-NO (EDHF) pathway alone unblocked compared with non-PG pathways (NO and EDHF) after training compared with the sedentary state in both controls and T2DM subjects (Figure 1).

However, as shown in Figure 2, no significant differences were found when suppression of maximally stimulated dorsal skin perfusion was examined as a percentage of suppression attributable directly to each vasodilator individually,

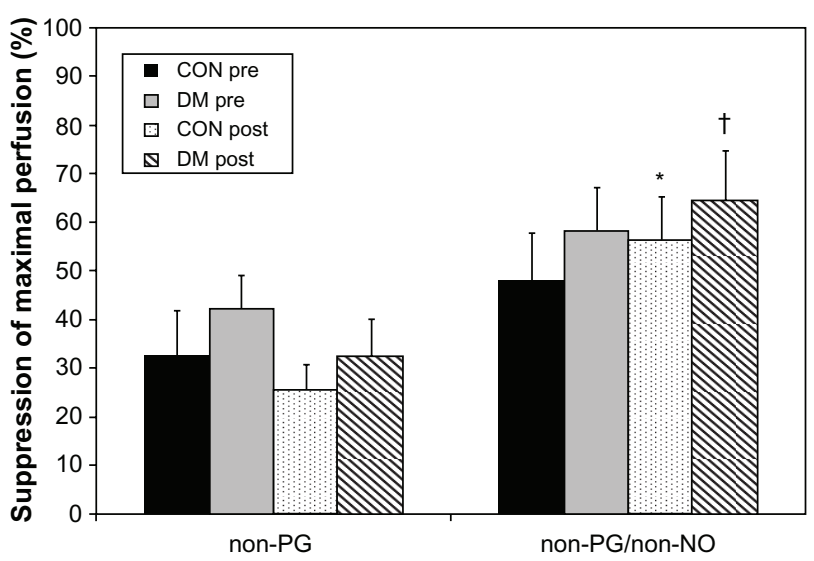

Figure I Suppression of maximally stimulated $\left(44^{\circ} \mathrm{C}\right)$ dorsal skin perfusion by subject group pre- and post-training. "Non-PG" conditions include oral ingestion of aspirin and systemic suppression of PG synthesis by aspirin (left foot), while "nonPG/non-NO" conditions have inhibition of PG by aspirin and add local blockade of $\mathrm{NO}$ via microdialysis infusion of L-NAME, a NO synthase inhibitor (right foot). These results include oral aspirin ingestion in all subjects and have not yet been compared with normative data collected without aspirin. Subject groups are differentiated by both diabetes and training status.

Notes: Black bars, control subjects pre-training; gray bars, diabetic subjects pretraining; spotted bars, control subjects post-training; striped bars, diabetic subjects post-training. ${ }^{*} P<0.05$ versus same group in non-PG; ${ }^{\dagger} P<0.01$ versus same group in non-PG.

Abbreviations: PG, prostaglandins; NO, nitric oxide; L-NAME, (N(G)-nitro-Iarginine methyl ester); CON, controls; DM, diabetes mellitus. 


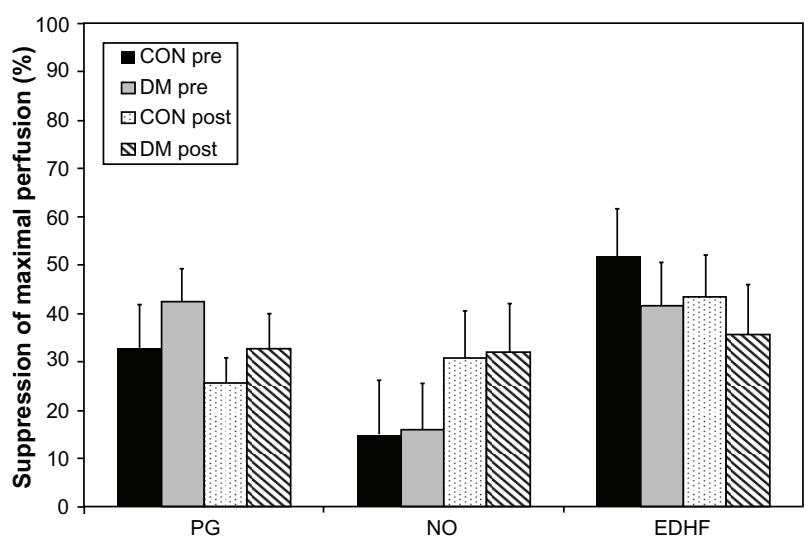

Figure 2 Suppression of maximally stimulated $\left(44^{\circ} \mathrm{C}\right)$ dorsal skin perfusion attributable directly to each vasodilator when grouped by diabetes and training status. These data are examined as a percent of suppression attributable directly to each vasodilator individually, determined using normative data collected previously in a similar subject group without oral aspirin ingestion. Subject groups are differentiated by both diabetes (DM versus controls) and pre- and post-training status.

Notes: Black bars, CON Pre, control subjects pre-training; gray bars, DM Pre, type 2 diabetic subjects pre-training; spotted bars, CON Post, control subjects posttraining; striped bars, DM Post, type 2 diabetic subjects post-training.

Abbreviations: PG, prostaglandins; NO, nitric oxide; EDHF, endothelial-derived hyperpolarizing factor; DM, diabetes mellitus; CON, controls; Pre, pretraining; Post, post-training.

determined using normative data collected previously in similar subject groups without oral aspirin ingestion. For these analyses, subject groups were differentiated by both diabetes (T2DM versus controls) and pre- and post-training status.

For subsequent analyses, the T2DM patients and controls were grouped due to nonsignificant differences between subject groups based on diabetes status. The relative contributions of NO, PG, and EDHF to maximal skin perfusion after six months of moderate aerobic training were determined using further analyses that again included the normative data without oral aspirin ingestion to block PG. In these analyses, the contributions of NO, PG, and EDHF were significantly increased, decreased, and unchanged by training, respectively, as shown in Figure 3. Using blockage of PG (with aspirin) or PG and NO (with aspirin and L-NAME), significantly less responsiveness of maximal perfusion was evident with the latter EDHF pathway only unblocked $(36.5 \% \pm 5.7 \%)$ posttraining compared with both $\mathrm{NO}$ and EDHF unblocked after training $(66.9 \% \pm 3.5 \% ; P<0.05)$.

\section{Discussion}

This study examined the importance and relative contributions of different vasodilatory compounds to dorsal foot skin perfusion during baseline and local heat-stimulated conditions in individuals with and without T2DM before and after six months of moderate, supervised aerobic training. We hypothesized that such training may alter the relative

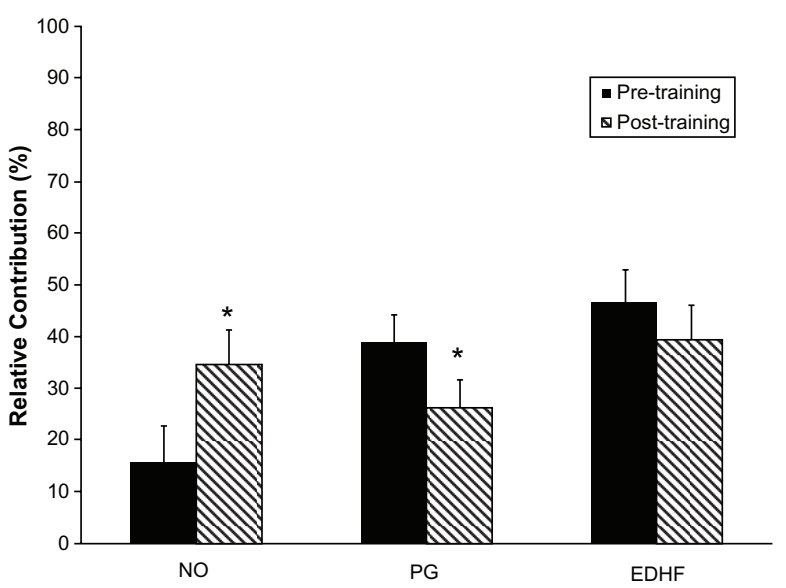

Figure 3 Relative contribution of three vasodilatory pathways in response to maximal dorsal foot skin perfusion. Due to nonsignificant differences between groups in suppression of maximally stimulated skin perfusion both pre- and posttraining, these data are presented with subject groups combined.

Notes: Black bars, pretraining ( $n=19, \mathrm{CON}$ and DM subjects combined); striped bars, post-training ( $\mathrm{n}=19, \mathrm{CON}$ and DM subjects combined). $* p<0.05$ pretraining versus post-training.

Abbreviations: CON, controls; DM, diabetes mellitus.

importance of PG, NO, and EDHF similarly to that previously observed in chronic exercisers, ie, increased PG, reduced EDHF, and unchanged NO contributions to stimulated perfusion responses. ${ }^{8}$

Contrary to our expectations, six months of training undertaken by sedentary individuals in the current study resulted instead in a significantly increased contribution to stimulated perfusion apparently coming from an NO mechanism, with reduced PG and unchanged EDHF. Consistent with these findings, Heidarianpour et $\mathrm{al}^{11}$ reported that 10 weeks of aerobic exercise training improves NO-dependent dilatation, possibly via the phosphoinositide 3-kinase pathway. However, when previously examining the effects of similar, moderate aerobic training done three times weekly for 10 weeks in participants with T2DM, we found no significant changes in measured levels of cutaneous interstitial NO in the dorsal foot during local heating despite differences in stimulated perfusion. ${ }^{12}$

Gooding et $\mathrm{al}^{1}$ investigated the vasodilatory mechanisms involved in maximal skin hyperemia in response to localized heating to greater $40^{\circ} \mathrm{C}$ or above, which is similar to the technique employed in the current study. In nondiabetic subjects, NO, but not PG, histamines, or an axon reflex, appeared to contribute most to the increase in blood flow in response to heating. Although NO levels are not necessarily altered by diabetes, ${ }^{3}$ defects in stimulated perfusion may result from a diminished local effectiveness of NO, particularly given that endothelium-dependent dilation in skin can be enhanced by eight weeks of moderate training and reversed by detraining. ${ }^{3,6,12}$ The current results are suggestive of a shift to a greater reliance on $\mathrm{NO}$ (via enhanced 
release and/or effectiveness) following relatively short-term participation in moderate aerobic exercise training and a lesser relative contribution of $\mathrm{PG}$, although more chronic participation (lasting a year or more) may later increase the importance of PG. ${ }^{8}$ The current study design did not allow for a direct measurement of NO to confirm that it remained unchanged, but all of our prior research supports this conclusion in similarly-aged diabetic and nondiabetic populations studied. ${ }^{12}$

Perfusion responses to such localized heating are largely controlled by small C-fiber nociceptors, ${ }^{13}$ and in the skin of diabetic subjects, significant C-fiber impairment and attenuated NO-mediated skin vasodilation have been reported. ${ }^{14}$ The defect in skin vasodilation is detectable before the development of diabetes and is partially correctable with insulin sensitizers, may be associated with $\mathrm{C}$-fiber dysfunction (ie, the dermal neurovascular unit), coexists with variables of the insulin resistance syndrome, and is partially correctable with insulin sensitizers. This defect may involve an imbalance among NO, substance $\mathrm{P}$, calcitonin gene-related peptide, angiotensin II, endothelin, and other vasodilatory or vasoconstrictive compounds., ${ }^{40}$ The participants in the current study were all studied during maximal stimulated conditions with systemic blockade of PG via oral aspirin ingestion. Therefore, it is not possible to determine whether this underlying defect was present. However, the study only included subjects with relatively good diabetes control and excluded those with severe diabetes-related complications (including those with severe peripheral neuropathy in whom the defect is often most pronounced), making it unlikely that this defect impacted the current findings. In addition, when present in diabetic subjects in previous studies, ${ }^{6,7}$ this defect did not appear to impact significantly on NO levels or maximally stimulated skin perfusion in the dorsal foot.

In healthy subjects, localized heat appears to stimulate an endothelial NO synthase response that generates NO to vasodilate skin, while neuronal NO synthase mediates more of the reflex responses to whole body heat stress. ${ }^{15,16}$ The results of the present study examining dorsal foot skin perfusion concur with these observations related to the effects of localized heating on forearm skin responses. With regard to the interactions of $\mathrm{NO}$ and $\mathrm{PG}$ on skin vasodilation, in healthy humans, chronic low-dose aspirin therapy attenuates the reflex cutaneous vasodilation during whole-body heating that is mediated through both the cyclooxygenase-dependent pathway of PG formation and NO synthase-dependent mechanisms. ${ }^{17}$ While vasodilation of skin in response to local heating is highly NO-dependent, it may also reflect the interaction of NO and PG. ${ }^{15,18}$ Cutaneous acetylcholine-mediated endothelium-dependent vasodilation has been reported to be highly NO-dependent and also strongly related to the interactions of NO with prostaglandins..$^{18}$ Thus, an interaction between these two vasodilatory compounds during localized heating of dorsal foot skin to $44^{\circ} \mathrm{C}$ is also a possibility, albeit an untested one, in this study.

Admittedly, the introduction of a microdialysis catheter into the cutaneal surface of the dorsal foot undeniably resulted in an immediate PG release and influx of interstitial fluids. Microdialysis probe insertion into the ventral forearm is known to cause a significant increase in skin blood flow, erythema, flare, and skin edema, ${ }^{19}$ and a 90-120 minute period of equilibration is required in human skin to allow the vascular reactions of the skin to stabilize. ${ }^{20}$ While it is unlikely that the skin perfusion completely normalized after the probe's insertion in the current study, PG dissipation was likely to have been relatively rapid during the initial hour of stabilization. Skin perfusion returned largely back to normal values before baseline testing was started after an hour, and another hour elapsed before maximal heating to $44^{\circ} \mathrm{C}$ was initiated, thereby allowing 120 minutes after the probe insertion before the maximal responses were tested.

As for the EDHF pathway, it may play a larger role only when NO or PG is compromised, ${ }^{2}$ which NO did not appear to be in this study, and EDHF may serve as more of a backup mechanism, particularly with aging. ${ }^{21}$ Both NO and PG are known to play important roles in skeletal muscle blood flow regulation during moderate intensity exercise, and EDHF does not compensate for the impaired formation of NO and prostaglandins in muscle. ${ }^{22}$ The relevance of these findings to cutaneal responses has not been established. In the presence of a diabetic state, toe skin perfusion has been shown to increase less in response to PG analogs when the ankle brachial index is lower, ${ }^{23}$ which is also a possible factor affecting the current findings, but one that was not able to be investigated in the current study design.

A recent study of the effects of ruboxistaurin on skin microvascular blood flow evaluated the relationship between endothelial and neural control of this perfusion in diabetic patients with diabetic peripheral neuropathy. ${ }^{24}$ They found a correlation between C-fiber-mediated and endotheliumdependent cutaneous perfusion, suggesting that improvements in endothelial function may affect the microcirculation not only locally, but also via the neurovascular arcade. As for the potential effects of neuropathic changes in the present study, skin perfusion was similar between control and diabetic groups, and measures of both central and peripheral 
neuropathy were similar between subject groups, making neuropathic changes unlikely to have affected the results.

In conclusion, six months of moderate aerobic exercise training appears not to alter baseline or maximal dorsal foot skin perfusion responses via EDHF, but does affect release of or sensitivity to $\mathrm{NO}$ and $\mathrm{PG}$, regardless of diabetes status. Thus, it is likely that improvements in dorsal foot skin perfusion resulting from six months of moderate training involve a non-EDHF pathway, most probably NO for shortterm (including six months of training) and PG for longerterm (ie, in chronic exercise) adaptations. Moreover, these findings suggest that regular exercise participation may have a bigger impact on maximal skin perfusion than diabetes alone, and may counter the negative effect that T2DM can exert on perfusion, at least in dorsal foot skin.

\section{Acknowledgments}

This work was fully supported by a clinical research grant from the American Diabetes Association. We would also like to acknowledge Amanda L Boyd and Kathleen R Sharp for their assistance in collecting these data.

\section{Disclosure}

The authors report no conflict of interest in this work.

\section{References}

1. Gooding KM, Hannemann MM, Tooke JE, Clough GF, Shore AC. Maximum skin hyperaemia induced by local heating: Possible mechanisms. J Vasc Res. 2006;43:270-277.

2. Lenasi H, Struci M. The effect of nitric oxide synthase and cyclooxygenase inhibition on cutaneous microvascular reactivity. Eur J Appl Physiol. 2008;103:719-726.

3. Sokolnicki LA, Roberts SK, Wilkins BW, Basu A, Charkoudian N. Contribution of nitric oxide to cutaneous microvascular dilation in individuals with type 2 diabetes mellitus. Am J Physiol. 2007;292: E31-E38.

4. Vinik AI, Erbas T, Park TS, Pierce KK, Stansberry KB. Methods for evaluation of peripheral neurovascular dysfunction. Diabetes Technol Ther. 2001;3:29-50.

5. Wang JS. Effects of exercise training and detraining on cutaneous microvascular function in man: The regulatory role of endothelium-dependent dilation in skin vasculature. Eur J Appl Physiol. 2005;93:429-434.

6. Colberg SR, Stansberry KB, McNitt PM, Vinik AI. Chronic exercise is associated with enhanced cutaneous blood flow in type 2 diabetes. J Diabetes Complications. 2002;16:139-145.

7. Colberg SR, Parson HK, Holton DR, Nunnold T, Vinik AI. Cutaneous blood flow in type 2 diabetic individuals following an acute bout of maximal exercise. Diabetes Care. 2003;26:1883-1888.
8. Colberg SR, Azoury KR, Parson HK, Vinik AI. Exercise status affects skin perfusion via prostaglandin, nitric oxide, and EDHF pathways in diabetes. Microvasc Res. 2009;77:120-124.

9. Cameron NE, Cotter MA. Metabolic and vascular factors in the pathogenesis of diabetic neuropathy. Diabetes. 1997;46:S31-S37.

10. Vinik AI, Suwanwalaikorn S, Stansberry KB, Holland MT, McNitt PM, Colen LE. Quantitative measurement of cutaneous perception in diabetic neuropathy. Muscle Nerve. 1995;18:574-584.

11. Heidarianpour A, Hajizadeh S, Khoshbaten A, Niaki AG, Bigdili MR, Pourkhalili K. Effects of chronic exercise on endothelial dysfunction and insulin signaling of cutaneous microvascular in streptozotocin-induced diabetic rats. Eur J Cardiovasc Prev Rehabil. 2007;14:746-752.

12. Colberg SR, Parson HK, Nunnold T, Holton DR, Swain DP, Vinik AI. Change in cutaneous perfusion following 10 weeks of aerobic training in type 2 diabetes. J Diabetes Complications. 2005;19:276-283.

13. Pergola PE, Kellogg DL, Johnson JM, Kosiba WA, Solomon DE. Role of sympathetic nerves in the vascular effects of local temperature in human forearm skin. Am J Physiol. 1995;265:H785-H792.

14. Kilo S, Berghoff M, Hilz M, Freeman R. Neural and endothelial control of microcirculation in diabetic peripheral neuropathy. Neurology. 2000;54:1246-1252.

15. Kellogg DL Jr, Zhao JL, Wu Y. Endothelial nitric oxide synthase control mechanisms in the cutaneous vasculature of humans in vivo. Am J Physiol. 2008;295:H123-H129.

16. Kellogg DL Jr, Zhao JL, Wu Y. Neuronal nitric oxide synthase control mechanisms in the cutaneous vasculature of humans in vivo. J Physiol. 2008;586:847-857.

17. Holowatz LA, Kenney WL. Chronic low-dose aspirin therapy attenuates reflex cutaneous vasodilation in middle-aged humans. J Appl Physiol. 2009; 106:500-505.

18. Medow MS, Glover JL, Stewart JM. Nitric oxide and prostaglandin inhibition during acetylcholine-mediated cutaneous vasodilation in humans. Microcirculation. 2008;15:569-579.

19. Anderson C, Andersson T, Wardell K. Changes in skin circulation after insertion of a microdialysis probe visualized by laser Doppler perfusion imaging. J Invest Dermatol. 1994;102:807-811.

20. Groth L, Serup J. Cutaneous microdialysis in man: Effects of needle insertion trauma and anaesthesia on skin perfusion, erythema and skin thickness. Acta Dermatol. 1998;78:5-9.

21. Gaubert ML, Sigaudo-Roussel D, Tartas M, Berrut G, Saumet JL, Fromy B. Endothelium-derived hyperpolarizing factor as an in vivo back-up mechanism in the cutaneous microcirculation in old mice. J Physiol. 2007;585:617-626.

22. Mortensen SP, González-Alonso J, Damsgaard R, Saltin B, Hellsten Y. Inhibition of nitric oxide and prostaglandins, but not endothelial-derived hyperpolarizing factors, reduces blood flow and aerobic energy turnover in the exercising human leg. $J$ Physiol. 2007;581:853-861.

23. Aso Y, Tayama K, Takanashi K, Inukai T, Takemura Y. Nitric oxide and prostaglandin inhibition during acetylcholine-mediated cutaneous vasodilation in humans. Microcirculation. 2001;50:568-572.

24. Brooks B, Delaney-Robinson C, Molyneaux L, Yue DK. Endothelial and neural regulation of skin microvascular blood flow in patients with diabetic peripheral neuropathy: Effect of treatment with the isoformspecific protein kinase C beta inhibitor, ruboxistaurin. $J$ Diabetes Complications. 2008;22:88-95.

\section{Publish your work in this journal}

Diabetes, Metabolic Syndrome and Obesity: Targets and Therapy is an international, peer-reviewed open-access journal committed to the rapid publication of the latest laboratory and clinical findings in the fields of diabetes, metabolic syndrome and obesity research. Original research, review, case reports, hypothesis formation, expert opinion and commentaries are all considered for publication. The manuscript management system is completely online and includes a very quick and fair peer-review system, which is all easy to use. Visit $\mathrm{http}: / / \mathrm{www}$. dovepress.com/testimonials.php to read real quotes from published authors. 